\title{
Triangular Metallic Gratings for High Efficiency Thin Film Solar Cells
}

\author{
Enes Battal ${ }^{1}$, Alper Taha Yoğurt ${ }^{1}$, Levent Erdal Aygun ${ }^{1}$ and Ali Kemal Okyay ${ }^{1,2}$ \\ ${ }^{1}$ Bilkent University, Department of Electrical and Electronics Engineering, Bilkent, TR-06800, Ankara, Turkey \\ ${ }^{2}$ UNAM-Institute of Materials Science and Nanotechnology, Bilkent University, TR-06800, Ankara, Turkey \\ *Tel:+90 (312) 290 1557, e-mail: aokyay@bilkent.edu.tr
}

\begin{abstract}
Our design of novel nanometallic structure integrated with photovoltaic devices provides polarization insensitive, broadband and significantly high absorptivity enhancement. This structure attains absorptivities higher than compared to similar thickness of Silicon solar cells with gratings.
\end{abstract}

\section{INTRODUCTION}

High efficiency and low cost photovoltaic (PV) devices are sought after to address energy and environmental challenges. There is a growing interest in thin-film PV devices, for meeting those challenges, however, thin active layers result in low light harvesting efficiency. Plasmonic effects can be utilized to improve light absorption in the thin active area, therefore, excitation of surface plasmon polariton (SPP) modes and localized surface plasmons (LSP) through integration of metallic gratings with thin-film organic and inorganic semiconductor based PV devices have been widely investigated in the recent literature [1-3]. For inorganic solar cells, rectangular metallic gratings placed on the top [4,5], at the bottom [6] and buried into the active layer [7] have been reported. In this work, we investigate, for the first time, triangular-prism shaped gratings integrated with thin-film inorganic PV devices for improved absorption efficiency.

Triangular-prism shaped nanometallic gratings at the bottom of the absorbing layer are computationally studied. Finite difference time domain analysis is used to compute field profiles using Lumerical FDTD Solutions [8]. Unlike many earlier reports, we achieve improved absorptivity under both transverse-electric (TE) and transverse-magnetic (TM) polarized illumination. We observe $48.5 \%$ overall absorbtivity enhancement with our optimal design (Fig. 1a) for the complete solar spectrum, compared to the reference structure (Fig. 1b), with a metallic back reflector (no grating).

\section{SiMULATION}

Our design assumes an amorphous-Silicon $(\alpha-\mathrm{Si})$ absorbing layer $\left(\mathrm{t}_{\alpha-\mathrm{Si}}=100 \mathrm{~nm}\right)$ with an indium tin oxide
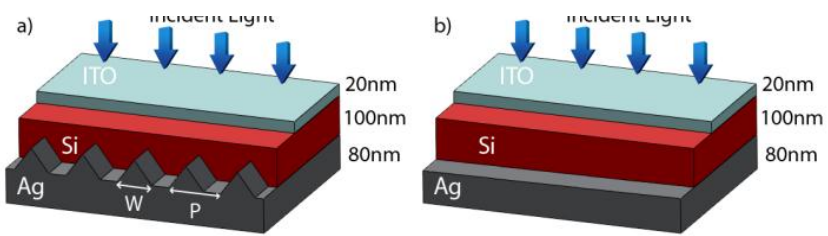

Fig. 1. a) Metallic gratings integrated solar cell structure b) Reference Structure (without gratings)
(ITO) $\left(\mathrm{t}_{\mathrm{ITO}}=20 \mathrm{~nm}\right)$ top contact for illumination and a silver (Ag) bottom electrode $\left(\mathrm{t}_{A \mathrm{~g}}=80 \mathrm{~nm}\right)$ with triangular gratings penetrating $\alpha-\mathrm{Si}$ layer. An isosceles triangular shape is assumed that can be produced from a template of anisotropic wet etched Silicon. The reference structure is without gratings.

Experimentally obtained optical constants for $\mathrm{Si}, \mathrm{Ag}$ [9] and ITO [10] are used in computations. We consider both TM and $T E$ polarized AM1.5G illumination. The absorptivity is calculated by $A=w \times \operatorname{Im}(\varepsilon) \oint|E|^{2} d V$, where $E$ is the electric field intensity, $V$ is the volume where the absorptivity is calculated and $\varepsilon$ is the dielectric constant of the active layer. We calculate total absorptivity as $\mathrm{A}_{\mathrm{TOTAL}}=\left(\mathrm{A}_{\mathrm{TM}}+\mathrm{A}_{\mathrm{TE}}\right) / 2$. The absorptivity enhancement is defined as the percentage increase of absorptivity $\left(\mathrm{A}_{\mathrm{INC}}\right)$ compared to the reference structure $\left(\mathrm{A}_{\mathrm{REF}}\right)$

\section{RESULTS AND ANALYSIS}

Our optimum design exhibits up to $23.6 \%$ absorptivity in AM1.5G while that for the reference device is $15.9 \%$. This result is above the previous reports of $100 \mathrm{~nm}$ and thicker Silicon active layer devices with single layer gratings $[4,6]$. The optimum design has $135 \mathrm{~nm}$ triangle base width and $470 \mathrm{~nm}$ period gratings corresponding to point A in Fig. 2.

In Fig. 3a, we observe the excitation of three different SPP modes for different grating period yielding strong absorptivity enhancement when $w=135 \mathrm{~nm}$ under TM-polarized illumination. In this figure, $\mathrm{S} 1, \mathrm{~S} 2$ and $\mathrm{S} 3$ represent the first, the second and the third order SPP modes. In addition, the excitation of LSPs is yet another mechanism of absorptivity enhancement due to the sharp corners of the triangular shaped grating structure. In Fig. 3b, increased absorption due to waveguide modes (WM) for $T E$-polarized light is shown, exhibiting red-shift with

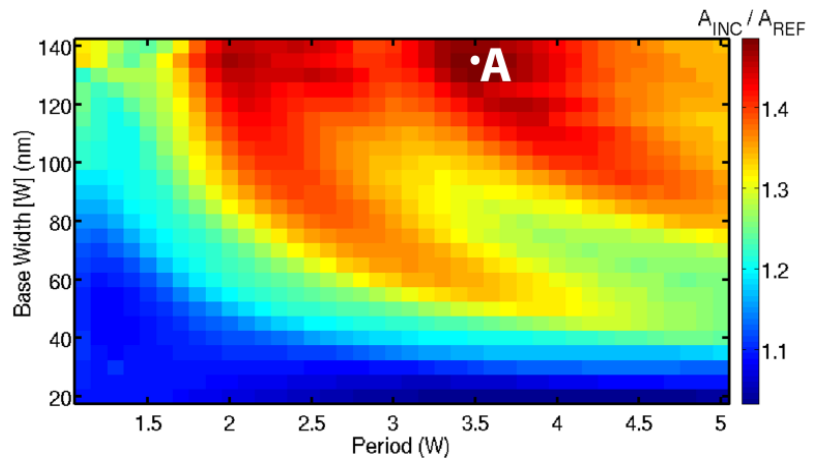

Fig. 2. Absorptivity enhancement as a function of base width $(w)(\mathrm{nm})$ and periodicity $(P)$, expressed in terms of multiples of $w$. 

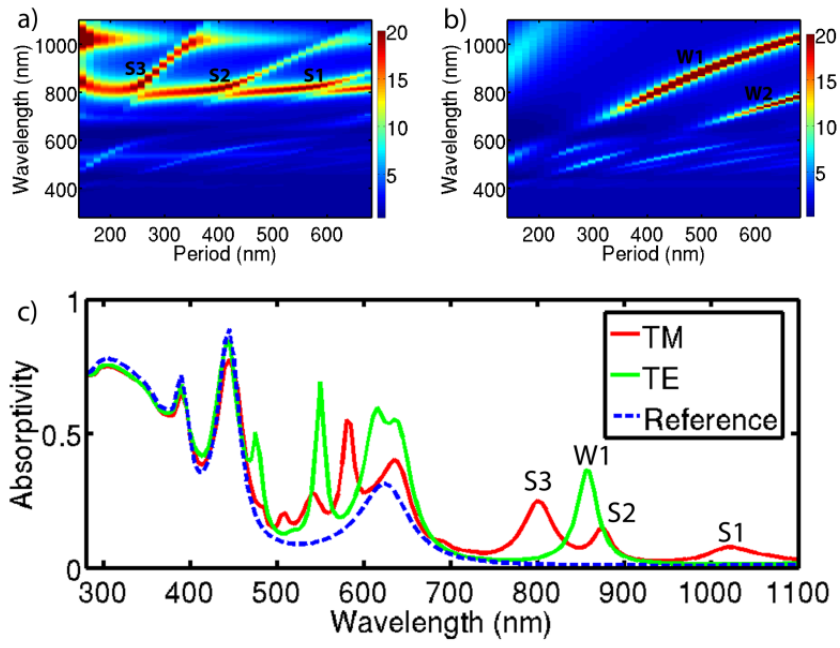

Fig 3. Absorptivity enhancement as a function of wavelength and periodicity for a) TM and b) TE polarized light when $w=135 \mathrm{~nm}$. c) Absorption as a function of wavelength for $w=135 \mathrm{~nm}$ and $P=470 \mathrm{~nm}$.

increasing grating period. There is also a comparably weaker contribution from Fabry-Perot resonances for shorter wavelengths. The combined effects of LSP and Fabry-Perott resonances, SPP and WM provide polarization insensitive, broadband and significantly high absorptivity enhancement.

In Fig. 3c, absorptivity enhancement vs illuminating wavelength is plotted for both $T E$ and $T M$ polarized light. We investigate the contribution of enhancement mechanisms at the wavelengths where absorption is strongly enhanced. At $\lambda=$ $800 \mathrm{~nm}, 875 \mathrm{~nm}$ and $1020 \mathrm{~nm}$, SPP modes S3, S2 and S1 are dominant, respectively, for $T M$-polarized illumination. The absorptivity characteristic for $\lambda>750 \mathrm{~nm}$ can be explained by the superposition of the three SPP modes, even though contributions from consecutive SPP modes are diminishing at longer wavelengths. The electric field intensity profile of the optimum structure at strongest SPP mode resonance, S3, is shown in Fig. 4a. The peaks observed at 510-nm and 540-nm under $T M$-polarization depends on the excitation of LSPs at the top corner of triangle However, the peak at $\lambda=585 \mathrm{~nm}$ is due to coupling of Fabry-Perot and LSP resonances, which is shown in Fig. 4b. For $T E$-polarized light, the resonance at the wavelength of $850 \mathrm{~nm}$ is directly addressed to excitation of waveguide mode which can be observed in Fig. 4c. Fig. 4d also shows the electric field profile for other peak observed under TE illumination.

Absorption behavior of the reference device and our design closely follow each other for $\lambda<450 \mathrm{~nm}$, for both polarizations. This behavior is attributed to the metallic nanograting being placed at the bottom of the active layer, avoiding ohmic losses of silver.
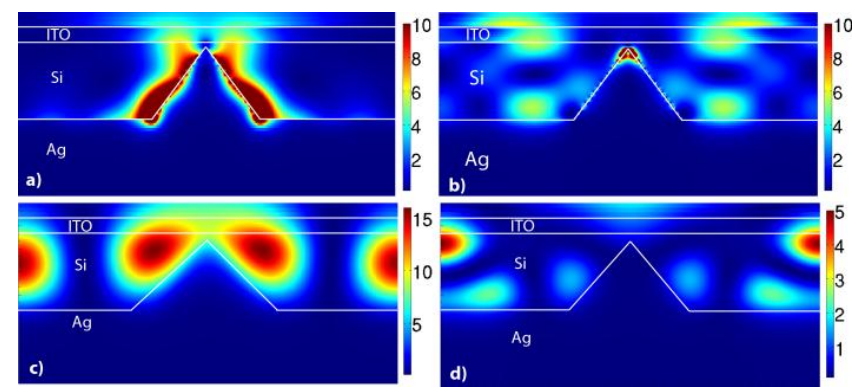

Fig. 4. E-field profile of the best structure at a) $\lambda=800 \mathrm{~nm}$ b) $\lambda=585 \mathrm{~nm}$ under TM polarization illumination and c) at $\lambda=850 \mathrm{~nm}$ and d) at $\lambda=550 \mathrm{~nm}$ under TE illumination

\section{CONCLUSION}

We demonstrate the design of a novel nanometallic structure integrated with photovoltaic devices which takes advantage of multiple SPP modes, LSPs, waveguide modes and Fabry-Perot resonances. This structure attains absorptivities as high as $23.6 \%$ under AM1.5G illumination, higher than the previously proposed Silicon solar cells with similar thickness and one dimensional single grating layer. We also briefly investigated the improvement mechanisms and angle dependency of the proposed structure.

\section{ACKNOWLEDGEMENTS}

This work was supported by TUBITAK 108E163, 109E044, EU FP7 PIOS 239444. The authors acknowledge TUBITAK BIDEP. The numerical calculations reported in this paper were performed at TUBITAK ULAKBIM, High Performance and Grid Computing Center (TR-Grid eInfrastructure).

\section{REFERENCES}

[1] Vivian E. Ferry, Marc A. Verschuuren, Hongbo B. T. Li, Ruud E. I. Schropp, Harry A. Atwater, and Albert Polman, Appl. Phys. Lett., 95, 183503 (2009)

[2] Mustafa Akin Sefunc, Ali Kemal Okyay, and Hilmi Volkan Demir, Appl. Phys. Lett. 98, 093117 (2011)

[3] Aimi Abass, Honghui Shen, Peter Bienstman, and Bjorn Maes, J. Appl. Phys. 109, 023111 (2011)

[4] Stephan Fahr, and Falk Lederer, J. Appl. Phys 104, 123102 (2008)

[5] Ragip A. Pala, Justin White, Edward Barnard, John Liu, and Mark L. Brongersma, Adv. Mater. 2009, 21, 3504-3509

[6] Wei Wang, Shaomin Wu, Kitt Reinhardt, Yalin Lu, and Shaochen Chen, Nano Lett. 2010, 10, 2012-2018

[7] Wenli Bai, Qiaoqiang Gan, Filbert Bartoli, Jing Zhang, Likang Cai, Yidong Huang, and Guofeng Song, Vol. 34, No. 23 / OPTICS LETTERS

[8] Lumerical Solutions Inc., Canada

[9] E. D. Palik, ed., "Handbook of Optical Constants of Solids," (Academic, Orlando, Fla., 1985).

[10] H. Hoppe, N. S. Sariciftci, and D. Meissner "Oprical constants of conjugated polymer/fullerene based bulk-heterojunction organic solar cells,” Mol. Cryst. Liq. Cryst. 385(1), 113-119 (2002). 